\title{
Histopathologic audit of hysterectomy specimens in a tertiary care hospital
}

\author{
Sujata Siwatch, Reetu Kundu, Harsh Mohan, Anju Huria ${ }^{1}$ \\ Sri Lanka Journal of Obstetrics and Gynaecology 2012; 34: 155-158
}

\begin{abstract}
Aims: To explore the pre-operative diagnosis, surgery performed and histopathological findings in teaching and non teaching hospitals in Chandigarh, India.
\end{abstract}

Methods: A retrospective analysis of preoperative diagnosis made available to the pathologist and the final histopathologic findings was done for age, indication of hysterectomy, type of surgery, hospital setting where surgery was performed and histopathology including gross and microscopic findings of the specimens.

Results: A total of 1,270 specimens were analyzed. More hysterectomies for malignant indications were done in teaching than in non teaching hospitals $(\mathrm{p}<0.001)$. One or both ovaries were removed in $46 \%$ of women undergoing hysterectomy in teaching hospitals and $54.3 \%$ in non teaching hospitals. Majority of the women were $30-45$ years of age. Fibroid uterus was the commonest indication (246 cases, $40 \%$ ) followed by prolapsed uterus (141 cases, $23 \%$ ) and menstrual dysfunction (117 cases, 19\%). Majority of the verifiable causes of hysterectomy were confirmed on histopathology. Besides, various incidental findings were noted in majority of the specimens.

Conclusions: There were significantly fewer hysterectomies for malignancy and a higher rate of oophorectomies with hysterectomy in non teaching than in teaching hospitals. Regular careful audit and review can help in improving the quality of health care in our country.

Key words: Hysterectomy, pathology, medical audit

\footnotetext{
${ }^{1}$ Government Medical College and Hospital, Sector32, Chandigarh, India.

Correspondence: Sujata Siwatch

E-mail: siwatch1@yahoo.com
}

\section{Introduction}

The lifetime risk of hysterectomies ranges from $20 \%$ to $35 \%{ }^{1-3}$. With the surge of medicolegal law suits, risk of premature surgical menopause after hysterectomy, surgical and anaesthetic risks involved and availability of alternative non invasive management options, every effort should be made to avoid hysterectomy wherever possible. Clinico-pathological audit of hysterectomies can help us define and improve our standards of diagnosis and justification of the hysterectomies performed. The aims of this study were to explore the pre-operative diagnosis, surgery performed and histopathologic findings in teaching and non teaching hospitals in Chandigarh, India.

\section{Materials and methods}

This was a retrospective audit. The pre-operative diagnosis and histopathologic data of all consecutive specimens of hysterectomies received in the Department of Pathology, Government Medical College and Hospital, Chandigarh, India from January 2009 to August 2011 were collected and analysed. The specimens were received from 3 main settingsGovernment Medical College Hospital, Government non-teaching service hospitals and private organisations/setups in the city. Clinical data collected included age, indication of hysterectomy and histopathologic data included gross and microscopic findings of the specimens i.e. uterus, adnexa and other samples sent taken during the surgery. Attempts were made to complete missing data from the hospital data where feasible. Data was analysed and statistical analysis done using SPSS version 11.

\section{Results}

During this period, a total of 1,270 hysterectomy specimens were received. These cases were almost equally distributed between those received from Government teaching hospitals (588 cases, $49.9 \%$ ) and those from non teaching Government/Public sector service hospitals and private organisations/setups (582 cases, 50.5\%). More hysterectomies for benign indications were done in non teaching hospitals than in teaching hospitals $(p=0.292)$ while in teaching 
hospitals more malignancies were operated $(p=$ $<0.001$ ). However, more panhysterectomies were performed in non teaching hospitals $(p=0.2131)$ and vice versa for total hysterectomies $(p<0.0003)$. Total hysterectomies with one adnexa and subtotal hysterectomies were also significantly higher in non teaching than in teaching hospitals ( $p=0.0004$ and 0.008 respectively). The mean age of the women undergoing all types of hysterectomies was $46.2 \pm 3.2$ years (19-79 years). Table 1 shows the distribution of preoperative indications and type of hysterectomies i.e. with or without removal of ovaries in various age groups. The mean age of women undergoing panhysterectomy was 47.2 years and the commonest indication for panhysterectomy was fibroid uterus. Overall, preoperative indications in 645 cases (50.7\%) were histopathologically verifiable while in 297 cases $(22.6 \%)$ histopathologic findings were suggestive of the indication of surgery e.g. surface keratinization in prolapse. Preoperative diagnosis was verified by histopathologic examination in 542 cases (84\%). No pathology was found in $3.9 \%$ of specimens, the commonest preoperative diagnosis in these cases was prolapsed uterus. Incidental findings and secondary changes observed at histopathology are detailed in Table 2.

Table 1. Distribution of cases of hysterectomy

Teaching hospitals $(n=628,49.4 \%)$

\begin{tabular}{lllllllll}
\hline $\begin{array}{l}\text { Indication } \\
\text { Age } \\
\text { (years) }\end{array}$ & Benign & Malignant & $\begin{array}{l}\text { Life } \\
\text { saving }\end{array}$ & $\begin{array}{l}\text { Most } \\
\text { common }\end{array}$ & TH & PAN & $\begin{array}{l}\text { TH and } \\
\text { one adnexa }\end{array}$ & $\begin{array}{l}\text { Subtotal } \\
\text { hysterectomy }\end{array}$ \\
\hline$<30$ & 3 & 1 & 8 & PPH & 5 & 1 & 7 & 1 \\
$30-45$ & 268 & 22 & 1 & Fibroid & 179 & 87 & 12 & 1 \\
$45-60$ & 217 & 30 & - & Fibroid & 111 & 155 & 6 & - \\
$>60$ & 47 & 17 & - & Prolapse & 44 & 17 & 2 & - \\
\hline
\end{tabular}

Non teaching hospitals $(n=642,50.5 \%)$

\begin{tabular}{lllllllll}
\hline $\begin{array}{l}\text { Age } \\
\text { (years) }\end{array}$ & Benign & Malignant & $\begin{array}{l}\text { Life } \\
\text { saving }\end{array}$ & $\begin{array}{l}\text { Most } \\
\text { common }\end{array}$ & TH & PAN & $\begin{array}{l}\text { TH and } \\
\text { one adnexa }\end{array}$ & $\begin{array}{l}\text { Subtotal } \\
\text { hysterectomy }\end{array}$ \\
\hline$<30$ & 2 & 2 & 4 & PPH & 1 & 2 & 3 & 2 \\
$30-45$ & 331 & 15 & 1 & Fibroid & 184 & 142 & 55 & 10 \\
$45-60$ & 191 & 6 & - & Fibroid & 80 & 137 & 2 & - \\
$>60$ & 36 & 4 & - & Prolapse & 17 & 7 & - & - \\
\hline
\end{tabular}

Table 2. Incidental findings and secondary changes observed on histopathology

\begin{tabular}{ll}
\hline Incidental histopathology findings and secondary changes & Cases $(\%)$ \\
\hline Adenomyosis & $240(18.9 \%)$ \\
Endometrial polyp & $47(3.7 \%)$ \\
Endometriotic cyst & $17(1.34 \%)$ \\
Senile cystic change & $12(0.94 \%)$ \\
Endometrial hyperplasia/ carcinoma & $11(0.87 \%)$ \\
Paratubal cyst & $7(0.55 \%)$ \\
Cervical premalignant changes (HSIL/ CIS) & $6(0.47 \%)$ \\
Chronic endometritis & $4(0.31 \%)$ \\
Lipoleiomyoma & $3(0.24 \%)$ \\
Hydrosalpinx/ salpingitis/ SIN & $1(0.08 \%)$ \\
\hline
\end{tabular}


Fibroid uterus was the commonest indication of hysterectomy (499 cases, 39\%). Fibroids were multiple in 319 cases $(60.9 \%)$. Their size varied from microscopic $(0.5 \mathrm{~cm})$ to as large as $25 \mathrm{~cm}$ diameter: $51.5 \%$ were less than $2 \mathrm{~cm}, 27.4 \%$ were $2-5 \mathrm{~cm}$ and $22.4 \%$ were $>5 \mathrm{~cm}$ in size. Their location was intramural $(72.9 \%)$, subserosal $(22 \%)$, submucosal $(3 \%)$ and at other sites such as broad ligament and cervix (2.2\%). The most common associated finding was adenomyosis. Hyaline change was seen in $57 \%$ of all fibroids.

Prolapsed uterus was indication for hysterectomy in 287 cases $(22.6 \%)$. The mean age of these women was 50 years. Total hysterectomy was done in most cases, with only 5 cases undergoing removal of one or both ovaries. Though prolapsed uterus is a clinical diagnosis, surface keratinization and atrophic changes associated with uterine prolapse were seen in $68.2 \%$ and $12.5 \%$ of specimens respectively.

Menstrual problems, most commonly menorrhagia and dysmenorrhoea, formed the indication for 235 of hysterectomies $(18.5 \%)$. The mean age of these cases was 41.6 years. Undiagnosed fibroids and adenomyosis were the commonest incidental findings in these patients. Hysterectomy for dysfunctional uterine bleeding (DUB) was done in 95 cases (7.5\%). Absence of any pathology confirmed the diagnosis of DUB in only $18.8 \%$ cases whereas others were found to have fibroids, adenomyosis and even endometrial carcinoma. Endometriosis was the indication in eight cases of hysterectomy. Pelvic/tubal/adnexal disease was noted in five cases whereas four specimens had ovarian endometriosis. The most common associated pathology was fibroid uterus.

Hysterectomy was done for abdominal/pelvic masses in 48 cases (3.7\%). On histopathology, 24\% were benign masses, $2 \%$ were borderline ovarian and $21 \%$ were malignant ovarian tumors. The commonest histopathologic diagnoses were serous cystadenomas and mucinous cystadenomas (27\% each). Panhysterectomy was done in all patients with adenexal masses. Hysterectomy for malignancies was done in 45 cases: ovarian in $38 \%$, endometrial in $42 \%$ and cervical in $20 \%$. Panhysterectomy was done in $95 \%$ and hysterectomy with unilateral oophrectomy in 5\% cases. Premalignant changes of the cervix were seen in 5 cases, 4 of which were incidental.

Postmenopausal bleeding was the indication for hysterectomy in 29 patients $(1.8 \%)$. The average age was 58 years. The causes included endometrial hyperplasia and endometrial polyp (5 cases each), carcinoma endometrium, carcinoma ovary, adenomyosis and prolapse uterus (4 each) and no findings in 3 cases.
Pregnancy-related indications for hysterectomy were seen in 14 cases $(1.1 \%)$. These included postpartum haemorrhage in 7 , adherent placenta in 4 , rupture uterus in 2 and uterine perforation in 1 case. The mean age of this group was 26.7 years.

\section{Discussion}

The indications for hysterectomies in non teaching service hospitals were frequently for benign conditions, yet the rate of panhysterectomy was higher. This fact emphasizes the need or regular audit of indications of hysterectomy and more comprehensive registry of number of hysterectomies which would help analyzing and reporting hysterectomies. However, the prevalence of subtotal hysterectomy in our study is low $(1.1 \%)$ as compared to $20.9 \%$ reported by Jacobson in $2003^{4}$.

Analyzing the indications for hysterectomy with the pathologic/surgical findings can help recognize malpractice and lacunae in the knowledge or training of health care service providers or non availability of newer alternatives for hysterectomy. The reporting of all hysterectomies should be made mandatory and the audit results used only for improvement in quality of health services and not for medico legal issues. In the absence of requirement of revalidation, the audit would help recognize the unmet need for planning trainings of continued medical education and update in this era of ever changing technologies and rising medico legal litigation. Implementing targeted remedial actions would help in offering more efficient and cost effective alternatives to hysterectomy tailored to the individual needs of the patients.

The most common indication for hysterectomy in our study was fibroid uterus which is in agreement with other reports in literature ${ }^{5,6}$. Prolapse and menstrual disorders have also been reported as common indication $^{7,8}$ The indications were histopathologically verifiable in $50.7 \%$ cases and verified in $84 \%$ of these. Al-Nuaim et al reported a confirmation of preoperative diagnosis in $69 \%$ specimens ${ }^{7}$. However, in contrast, we did not include prolapsed uterus as a verifiable indication. Our study showed much fewer specimens with unremarkable pathology $(3.9 \%)$ in contrast to study by Jha et al $(38 \%)^{8}$. There were various incidental findings in a significant number of specimens which could have been detected pre-operatively. Adenomyosis was the most common finding missed preoperatively. Higher degree of suspicion and better technique may help in diagnosing the missed indications. The mention of all findings on histopathology request forms is important to correlate the pre and postoperative findings and justify the decision for hysterectomy. 
Our study is a retrospective study and is limited by the lack of complete documentation in many cases, bias of documentation of pre-operative diagnosis, bias of cases where histopathology had been requested, especially in the non teaching sector and the lack of information of other consequences leading to the extent of surgery. However, the large sample size, multicentric origin of the specimens and short time duration ensuring uniformity in the reporting of histopathologic specimens may increase the validity of the study.

\section{Conclusion}

There were significantly fewer hysterectomies for malignancy and a higher rate of oophrectomies with hysterectomy in non teaching service hospitals than in teaching hospital. The most common indication for the age group 30 - 60 years was fibroid uterus and for more than 60 years was prolapsed uterus. Majority of the verifiable causes of hysterectomy were confirmed on histopathology. Various incidental findings still presented in significant number of specimens. Regular careful audit can help in improving the quality of health care services in our country and providing safe and cost effective management options.

\section{References}

1. Sait K, Alkhattabi M, Boker A, Alhashemi J. Hysterectomy for benign conditions in a university hospital in Saudi Arabia. Ann Saudi Med 2008; 28(4): 282-6.

2. Spilsbury K, Semmens JB, Hammond I, Bolck A. Persistent high rates of hysterectomy in Western Australia: a population-based study of 83000 procedures over 23 years. BJOG 2006; 113(7): 804-9.

3. MacKenzie IZ, Naish C, Rees M, Manek S. 1170 consecutive hysterectomies: indications and pathology. J Br Menopause Soc 2004; 10(3): 108-12.

4. Jacobson GF, Shaber RE, Armstrong MA, Hung YY. Hysterectomy rates for benign indications. Obstet Gynecol 2006; 107(6): 1278-83.

5. Abe E, Omo-Aghoja LO. A decade of hysterectomy in a tertiary hospital in urban Niger-Delta region of Nigeria. Niger J Clin Pract 2008; 11(4): 359-63.

6. Leung PL, Tsang SW, Yuen PM. Quality Assurance Subcommittee in Obstetrics and Gynaecology, Hospital Authority, Hong Kong. An audit on hysterectomy for benign diseases in public hospitals in Hong Kong. Hong Kong Med J 2007; 13(3): 187-93.

7. Al-Nuaim LA, Esset J, Banu F, Chowdhury N. An appraisal of the appropriateness of the indications for hysterectomy. J Obstet Gynaecol 1997; 17(5): 461-4.

8. Jha R, Pant AD, Jha A, Adhikari RC, Sayami G. Histopathological analysis of hysterectomy specimens. J Nepal Med Assoc 2006; 45(163): 283-90. 